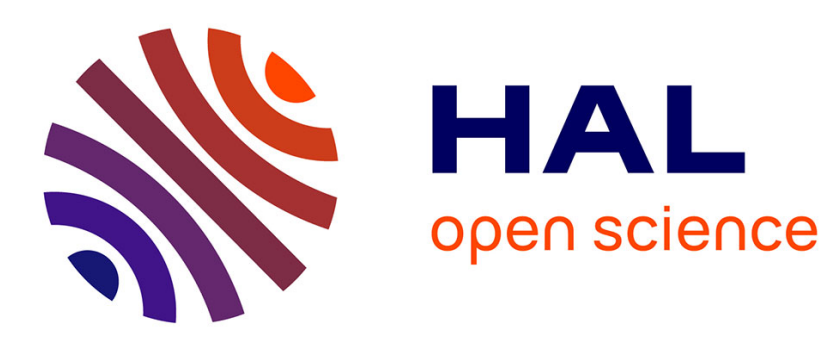

\title{
A Geometrical Approach for Power Optimization in Relay-Based Cellular Networks
}

Mattia Minelli, Maode Ma, Marceau Coupechoux, Philippe Godlewski

\section{To cite this version:}

Mattia Minelli, Maode Ma, Marceau Coupechoux, Philippe Godlewski. A Geometrical Approach for Power Optimization in Relay-Based Cellular Networks. IEEE National Conference on Communications (NCC), Feb 2014, Kanpur, India. pp.1-6. hal-00937203

\section{HAL Id: hal-00937203 https://hal-imt.archives-ouvertes.fr/hal-00937203}

Submitted on 28 Jan 2014

HAL is a multi-disciplinary open access archive for the deposit and dissemination of scientific research documents, whether they are published or not. The documents may come from teaching and research institutions in France or abroad, or from public or private research centers.
L'archive ouverte pluridisciplinaire HAL, est destinée au dépôt et à la diffusion de documents scientifiques de niveau recherche, publiés ou non, émanant des établissements d'enseignement et de recherche français ou étrangers, des laboratoires publics ou privés. 


\title{
A Geometrical Approach for Power Optimization in Relay-Based Cellular Networks
}

\author{
Mattia Minelli ${ }^{1,2}$, Maode $\mathrm{Ma}^{1}$, Marceau Coupechoux ${ }^{2}$, and Philippe Godlewski ${ }^{2}$ \\ ${ }^{1}$ Nanyang Technological University, Nanyang Avenue, Singapore \\ 2 Télécom ParisTech and CNRS LTCI, 46, rue Barrault, Paris, France
}

\begin{abstract}
Relays are considered as a feasible way to increase coverage and capacity of cellular networks. However, optimization of relay-enhanced networks is still a matter of research. This paper proposes a simple geometrical approach to derive the optimal long-term relay downlink transmit power with the aim of maximizing the average spectral efficiency in a relay-based cellular network. In our model, a User Equipment (UE) is served by the network node from which it receives the highest power. The spectral efficiency at the UE location is maximized when this serving node is also the one that provides the best Signal to Interference Ratio (SIR). Our approach thus consists in setting the relay node ( $R N)$ power such that its serving area and its SIR area coincide as much as possible. Results show that our algorithm provides a fine approximation of the optimal RN transmit power under a wide range of RN location scenarios and propagation parameters.
\end{abstract}

\section{INTRODUCTION}

The deployment of Relay Nodes (RN) is a central feature of next generation cellular networks (LTE-A and IEEE $802.16 \mathrm{j}$ ). In this paper, we consider decode-andforward non-cooperative RNs [1] and we focus on the downlink. Our goal is to optimize the RNs transmit power on the long-term in order to maximize the cell average spectral efficiency.

Several papers in the literature tackle the problem of optimal transmit power in relay-assisted cellular networks. In [2], authors come up with a convex weighted power minimization problem under rate, bandwidth and power constraints and give some insights on the optimal solution. In [3], a system capacity maximization problem is formulated under adaptive sub-channel, path and

Email addresses: mattia1@e.ntu.edu.sg (Mattia Minelli); emdma@ ntu.edu.sg (Maode Ma); coupecho@enst.fr (Marceau Coupechoux); godlewsk@enst.fr (Philippe Godlewski) power allocation. A heuristic is proposed to approximately solve the mixed integer programming problem. Authors of [4] maximize the system goodput with appropriate sub-band, power and rate allocation, while taking into account fairness and imperfect channel information. The main drawbacks of these approaches (and other in the literature, e.g. [5]) are the computational complexity and the induced protocol overhead. Proposed solutions indeed require to be regularly updated on a frame-byframe basis in order to adapt RN and Base-Station (BS) powers to variable traffic, UEs channel conditions, etc. On the contrary, setting a constant transmit power has the advantage of simplicity and is a common assumption in multi-cell performance evaluations [6], [7]. In this case, the need arises for optimizing capacity by appropriately setting, at the network deployment, the RN power for a long term.

In this paper, we thus derive an approximation of the long-term optimal $\mathrm{RN}$ transmit power. Our approach is based on matching the serving area of a RN (the set of locations served by the relay) with its SIR area (the area where the relay provides the best SIR) as closely as possible. We use the fluid model [8] as a means to approximate the average SIR. The implementation of our method improves network performance without requiring any additional protocols overhead. Moreover, no extra computational burden for BSs is involved, and RNs transmit power does not need to be regularly updated. We show that our algorithm yields accurate results, as compared to the actual optimal long-term RNs transmit power, in numerous simulation scenarios. Algorithm complexity decreases when fluid model is used to compute SIR, as well as results accuracy. This 

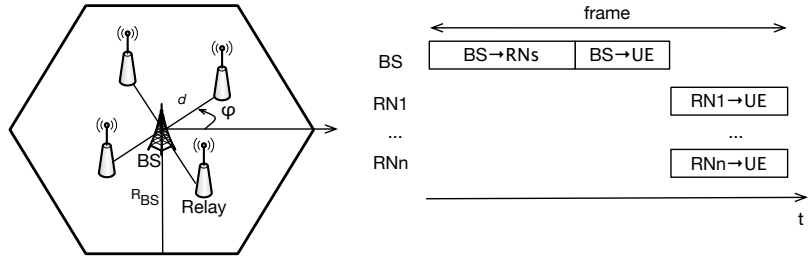

Fig. 1. Relay location (left) and radio resource partitioning (right).

tradeoff is exhaustively discussed.

The paper is organized as follows: Section II describes the system model. Section III formulates the optimization problem and Section IV presents our maximization approach. Section V gives numerical results, while Section VI concludes the paper.

\section{System Model}

We consider the downlink of a single-frequency hexagonal cellular network in an urban environment. Let the cell radius be less than $1 \mathrm{~km}$. One BS is located in the center of each cell. The BS is assisted by $N_{R N}$ $\mathrm{RN}$, placed on a circle of radius $d$ around the BS. $\mathrm{RN}$ are regularly spaced on the circle (see e.g. [2] [9]). Let $\phi$ be the angle shift of the first RN with respect to the horizontal axis (see Fig. 1, left). BSs and RNs transmit respectively with power $P_{B S}$ and $P_{R N}$, with $P_{R N}<P_{B S}$ [1]. The set of network nodes is denoted with $\mathcal{S}=\mathcal{S}_{R} \cup \mathcal{S}_{B}$, where $\mathcal{S}_{R}$ represents the set of RNs and $\mathcal{S}_{B}$ is the set of BSs of cardinality $\left|\mathcal{S}_{B}\right|=B$. Both $\mathrm{BS}$ and $\mathrm{RN}$ are equipped with a single omni-directional antenna and they transmit in division mode of operations, i.e., using orthogonal timefrequency resources (see e.g. [10]). An example of time division is shown in Fig. 1 (right).

\section{A. Serving and SIR areas}

Received power at location $z$ from node $i$ is given by:

$$
P_{i}(z)=P_{t x, i} K d_{i, z}^{-\eta},
$$

where $P_{t x, i} \in\left\{P_{B S} ; P_{R N}\right\}$ is the transmit power of $i$, $K$ is a constant, $d_{i, z}$ is the distance between $i$ and $z$ and $\eta$ is the propagation exponent. UEs are supposed to be distributed over the cell area according to a uniform probability distribution (homogeneous spatial Poisson process). UEs are served by the node (the best server) providing the highest received power as e.g. in [7]. We define the serving area of $i$ as the set of locations where $i$ is the serving station.
We consider thermal noise to be negligible compared to the sum of in-cell and out-of-cell interference, in agreement with [8], which starts from the same assumptions made in this paper. Hence, Signal to Interference Ratio (SIR) is chosen as radio channel performance measure. At location $z$, the SIR provided by node $i$ is given by:

$$
\Gamma_{i}(z)=\frac{P_{t x, i} K d_{i, z}^{-\eta}}{\sum_{k \in \mathcal{S}_{i} \backslash\{i\}} P_{t x, k} K d_{k, z}^{-\eta}}=\frac{d_{i, z}^{-\eta}}{\sum_{k \in \mathcal{S}_{i} \backslash\{i\}} d_{k, z}^{-\eta}},
$$

where $\mathcal{S}_{i}$ is the set of nodes of the same kind of $i$ (BS or $\mathrm{RN})$. We define the SIR area of $i$ as the set of locations where $i$ provides the best SIR.

Fig. 2 illustrates the notions of serving and SIR areas. It focuses on a BS $i$ and a $\mathrm{RN} j$ in a cell $\mathcal{A}_{c} . \mathcal{A}_{i j}^{P}\left(P_{R N}\right)$ denotes the area (delimited by the curve $\pi_{i j}\left(P_{R N}\right)$ ), where the power received by $i$ is higher than the one received by $j$. The serving area of $i$ is the intersection of all $\mathcal{A}_{i j}^{P}$, where $j$ is the index of a $\mathrm{RN}$ of the cell. $\mathcal{A}_{i j}^{S}$ is the area, where the SIR obtained from $i$ is higher than the SIR obtained from $j$ and the SIR area is the intersection of such areas in the cell.

According to (1) and (2), the SIR area is independent of the nodes transmit power, whereas the serving area depends on $P_{R N}$, and can be smaller or greater than the SIR area according to it. We denote $\mathcal{D}_{i j}\left(P_{R N}\right)$ the symmetric difference of the two sets in the cell, i.e.,

$$
\mathcal{D}_{i j}\left(P_{R N}\right) \triangleq \mathcal{A}_{c} \cap\left(\mathcal{A}_{i j}^{S} \triangle \mathcal{A}_{i j}^{P}\right)
$$

where the symmetric difference $\triangle$ is defined for any two sets $A$ and $B$ as follows: $A \triangle B \triangleq(A \backslash B) \cup(B \backslash A)$. Let $\mathcal{D}_{i} \triangleq \cup_{j \in \mathcal{S}_{R}} \mathcal{D}_{i j} . \mathcal{D}_{i}$ represents the area in the cell, where UEs are not served by the best station.

\section{B. Fluid Model}

Under our assumptions $\Gamma_{i}(z)$ can be approximated by means of the fluid model [8] for the regular hexagonal network formed by the BSs. The fluid model is a powerful tool for simplifying SIR formulas in a wireless network. The main idea is to replace a discrete set of transmitters by a continuum and thus transform discrete sums into integrals.

If $i \in \mathcal{S}_{B}$ and the network is large we have:

$$
\Gamma_{i}(z) \approx \frac{\eta-2}{2 \pi \rho_{B S} d_{i, z}^{\eta}\left(2 R_{B S}-d_{i, z}\right)^{2-\eta}},
$$

where $\rho_{B S}$ is the BS density and $R_{B S}$ is the half interBS distance. Strictly speaking, the fluid model is not 


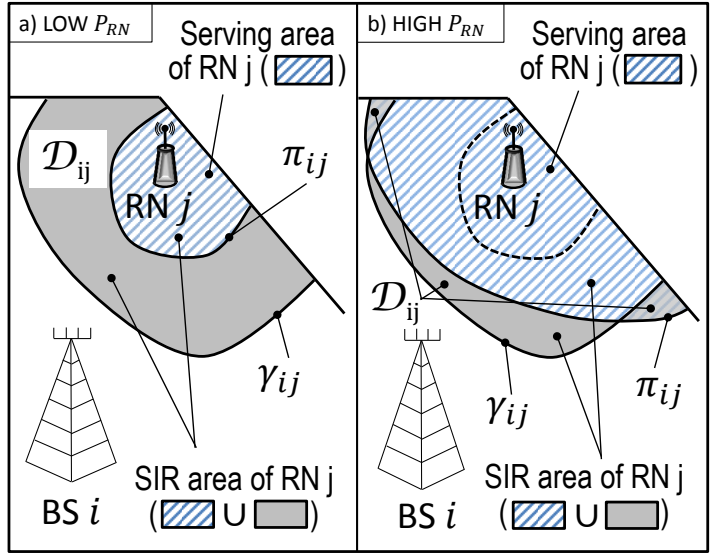

Fig. 2. SIR and serving areas of $\mathrm{RN} j$.

applicable to the generally non-regular $\mathrm{RN}$ network. However, we show that in a number of scenarios this method still provides a fair approximation of $\Gamma_{i}(z), i \in$ $\mathcal{S}_{R}$ (see Section $\mathrm{V}$ ) provided that $R_{B S}$ is replaced by $R_{R N}$, the minimum half inter-RN distance, and $\rho_{B S}$ by $\rho_{R N}$, the RN density.

\section{Spectral Efficiency}

The spectral efficiency at location $z$ is defined as follows:

$$
C\left(z, P_{R N}, P_{B S}\right)=\log _{2}\left(1+\Gamma_{b\left(z, P_{R N}, P_{B S}\right)}(z)\right),
$$

where $b\left(z, P_{R N}, P_{B S}\right)$ is the serving station of $z$ (as we focus on $P_{R N}$ optimization, the dependence on $P_{B S}$ is skipped in the following developments). We also define $C_{H}(z)$ as the spectral efficiency in $z$ associated to the station providing the best SIR in $z$.

\section{Problem Formulation}

Our aim is to maximize the cell average spectral efficiency on the downlink, via a proper tuning of the RN transmit power. The optimization is focused on a generic cell $\mathcal{A}_{c}$ of area $A_{c}$, centered at BS $i$. We formulate the problem as follows:

$$
P_{R N}^{*}=\underset{P_{R N}}{\operatorname{argmax}}\left\{\bar{C}\left(P_{R N}\right)\right\}
$$

under the constraint:

$$
0<P_{R N}<P_{B S}
$$

where $\bar{C}\left(P_{R N}\right)=\frac{1}{A_{c}} \int_{\mathcal{A}_{c}} C\left(z, P_{R N}\right) d z$ is the cell average spectral efficiency.

Ideally, the highest $\bar{C}\left(P_{R N}\right)$ is reached for $\mathcal{D}_{i} \equiv \varnothing$, i.e., when all UEs are served by the station which can guarantee them the highest SIR. However, this condition cannot be achieved in general, as there is normally no $P_{R N}$ for which $\mathcal{A}_{i j}^{P} \equiv \mathcal{A}_{i j}^{S}$.

Let us work out (6) as follows:

$$
\begin{aligned}
P_{R N}^{*} & =\underset{P_{R N}}{\operatorname{argmin}}\left\{-\bar{C}\left(P_{R N}\right)\right\} \\
& =\underset{P_{R N}}{\operatorname{argmin}}\left\{K-\bar{C}\left(P_{R N}\right)\right\} \\
& =\underset{P_{R N}}{\operatorname{argmin}}\left\{\frac{1}{A_{c}} \int_{\mathcal{A}_{c}}\left(C_{H}(z)-C\left(z, P_{R N}\right)\right) d z\right\},
\end{aligned}
$$

where $K \in \mathbb{R}$ is an arbitrary constant. The second equality is valid because $\frac{1}{A_{c}} \int_{\mathcal{A}_{c}} C_{H}(z) d z$ is a constant w.r.t. $P_{R N}$. Now, the difference in the integrand is zero outside $\mathcal{D}_{i}$. Hence (8) is equivalent to

$$
P_{R N}^{*}=\underset{P_{R N}}{\operatorname{argmin}}\left\{\frac{1}{A_{c}} \int_{\mathcal{D}_{i}}\left(C_{H}(z)-C\left(z, P_{R N}\right)\right) d z\right\} .
$$

Solving (9) requires finding the borders of $\mathcal{D}_{i}$ and computing the integral for every candidate value of $P_{R N}$. However, it is possible to restrict the range of $P_{R N}$ to be explored. This range being very tight, it is actually not even needed to evaluate the integral, as it will be shown in the next section. Solution is proposed for the simple system model hypothesized in this paper. However, our approach can still be applied with few modifications to more complex scenarios, i.e., when dealing with more complex path-loss or non-hexagonal BS network.

\section{Proposed Solution}

In this section, we first derive the borders of the area $\mathcal{D}_{i j}$ and then show that the spectral efficiency is first increasing and then decreasing with $P_{R N}$. This allows us to propose a fast algorithm to numerically solve the optimization problem.

\section{A. Derivation of $\pi_{i j}\left(P_{R N}\right)$ and $\gamma_{i j}$}

Let us again focus on a pair of $\mathrm{BS} i$ and $\mathrm{RN} j$ in a cell $\mathcal{A}_{c}$. The expression of $\pi_{i j}\left(P_{R N}\right)$ is found by equating the received powers from $i$ and $j$. With the axis system shown on Fig. 2 and using (1), we can write for $(x, y) \in$ $\mathcal{A}_{c}$ :

$$
\pi_{i j}\left(P_{R N}\right):\left(x-x_{\delta}\right)^{2}+\left(y-y_{\delta}\right)^{2}=r^{2},
$$

where $r=\sqrt{\Omega\left(x_{R N}(j)^{2}+y_{R N}(j)^{2}\right) /(\Omega-1)^{2}}, \Omega=$ $\left(P_{R N} / P_{B S}\right)^{2 / \eta}$ and $\left(x_{R N}(j), y_{R N}(j)\right)$ is the location 
of RN $j$. Hence, $\pi_{i j}\left(P_{R N}\right)$ is a part of a circle of radius $r$, increasing with $P_{R N}$. The circle is centered at $\left(x_{\delta}, y_{\delta}\right)=\left(x_{R N} /(1-\Omega), y_{R N} /(1-\Omega)\right)$, along the line connecting $i$ and $j$. It is easy to verify that increasing $P_{R N}$ has the effect shown on Fig. 2, i.e., the set $\mathcal{A}_{i j}^{P}$ is decreasing. Similarly to (10), $\gamma_{i j}$ is found by equating the SIR expressions related to $i$ and $j$. If we apply the fluid model, we obtain for $(x, y) \in \mathcal{A}_{c}$ :

$$
\gamma_{i j}: \frac{\rho_{B S}}{\rho_{R N}}\left(\frac{d_{i}(x, y)}{d_{j}(x, y)}\right)^{\eta}=\left(\frac{2 R_{R N}-d_{j}}{2 R_{B S}-d_{i}}\right)^{2-\eta},
$$

where $d_{i}(x, y)=\sqrt{x^{2}+y^{2}}$ and $d_{j}(x, y)=$ $\sqrt{\left(x-x_{R N}(j)\right)^{2}+\left(y-y_{R N}(j)\right)^{2}}$. If the fluid model does not provide a sufficient approximation, the SIR can be directly computed with (2). Note again that varying $P_{R N}$ does not affect the equation of $\gamma_{i j}$.

\section{B. Derivation of Bounds on $P_{R N}$ Search Range}

We see from Fig. 2 that starting from a low $\mathrm{RN}$ power and increasing its value has the effect to first decrease and then increase the area $\mathcal{D}_{i j}$, while average spectral efficiency first increases and then decreases. This intuition is formalized as follows.

Lemma 1: (i) Suppose that for every RN $j$ in the cell, $\mathcal{A}_{i j}^{S} \subseteq \mathcal{A}_{i j}^{P}\left(P_{R N}\right)$, then $\bar{C}\left(P_{R N}\right)$ is increasing in $P_{R N}$. (ii) Suppose that for every RN $j$ in the cell, $\mathcal{A}_{i j}^{P}\left(P_{R N}\right) \subseteq$ $\mathcal{A}_{i j}^{S}$, then $\bar{C}\left(P_{R N}\right)$ is decreasing in $P_{R N}$.

Proof: For (i) consider two RN powers $P_{2}>P_{1}$ for which the condition is valid. Using (10), it is easy to verify that $\mathcal{A}_{i j}^{P}\left(P_{2}\right) \subseteq \mathcal{A}_{i j}^{P}\left(P_{1}\right)$. Using now the definition of $\mathcal{D}_{i j}$ in (3) and the lemma first condition, we obtain $\mathcal{D}_{i j}\left(P_{2}\right) \subseteq \mathcal{D}_{i j}\left(P_{1}\right)$ for every $j$. For $P_{R N}=P_{2}$ integral (9) is hence computed on a subset of the area associated to $P_{1}$ and the integrand is positive, which implies $\bar{C}\left(P_{2}\right) \geqslant \bar{C}\left(P_{1}\right)$. For (ii) the proof is similar, except that now $\mathcal{D}_{i j}\left(P_{1}\right) \subseteq \mathcal{D}_{i j}\left(P_{2}\right)$.

According to lemmas $1, P_{R N}^{*}$ must thus lie between a lower and upper bound: $P_{R N}^{L} \leqslant P_{R N}^{*} \leqslant P_{R N}^{U}$, where $P_{R N}^{L}$ and $P_{R N}^{U}$ are respectively the highest and the lowest $P_{R N}$ for which the conditions $\mathcal{A}_{i j}^{S} \subseteq \mathcal{A}_{i j}^{P}\left(P_{R N}\right)$ and $\mathcal{A}_{i j}^{P}\left(P_{R N}\right) \subseteq \mathcal{A}_{i j}^{S}$ are valid for all $j$. Note that in general we cannot draw any conclusion about spectral efficiency when $P_{R N}^{L} \leqslant P_{R N} \leqslant P_{R N}^{H}$. Bounds can be interpreted as follows:

- $P_{R N}^{L}$ is the RN power for which $\mathcal{A}_{i j}^{S} \subseteq \mathcal{A}_{i j}^{P}\left(P_{R N}\right)$ and $\pi_{i j}\left(P_{R N}\right)$ is tangent to $\gamma_{i j}$ for some $j$.

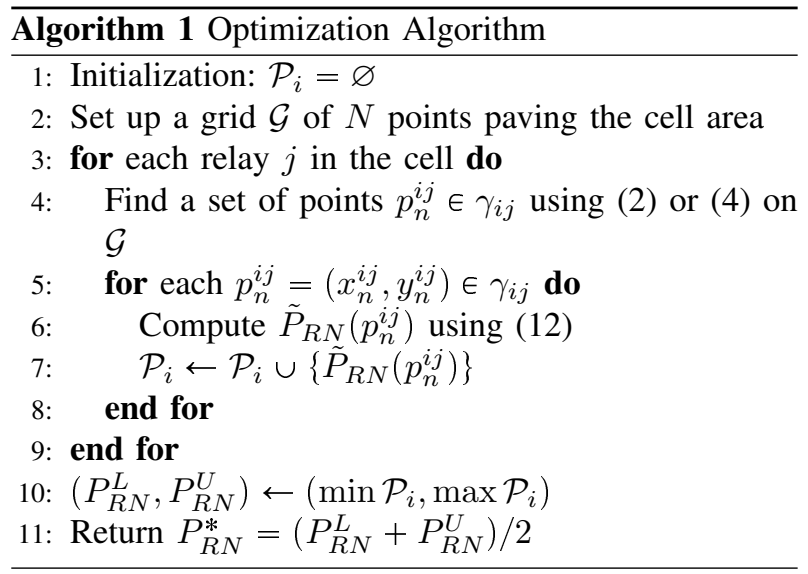

- $P_{R N}^{U}$ is the RN power for which $\mathcal{A}_{i j}^{P}\left(P_{R N}\right) \subseteq \mathcal{A}_{i j}^{S}$ and $\pi_{i j}\left(P_{R N}\right)$ is tangent to $\gamma_{i j}$ for some $j$.

The bounds cannot be derived analytically since (11) has no explicit solution. We can however obtain approximations in three steps:

1) We find numerically a set of points $\left\{p_{n}^{i j}\right\}_{n \in\left[1, N_{i j}\right]} \in \gamma_{i j}$. For that, we build a regular grid of $N$ points on the cell area and, using (2) or (4), we find the $p_{n}^{i j}$ by interpolation.

2) For each $p_{n}^{i j}=\left(x_{n}^{i j}, y_{n}^{i j}\right)$, we find the associated RN power $\tilde{P}_{R N}\left(p_{n}^{i j}\right)$, defined as the power for which $x_{n}^{i j}$ and $y_{n}^{i j}$ satisfy the equation of $\pi_{i j}\left(P_{R N}\right)$. It is found by substituting $x_{n}^{i j}$ and $y_{n}^{i j}$ into (10), and solving for $P_{R N}$, obtaining:

$$
\tilde{P}_{R N}\left(p_{n}^{i j}\right)=P_{B S}\left(l_{n j}^{2} / l_{n 0}^{2}\right)^{\frac{\eta}{2}},
$$

where $l_{n j}^{2}=\left(x_{n}^{i j}-x_{R N}(j)\right)^{2}+\left(y_{n}^{i j}-y_{R N}(j)\right)^{2}$ is the squared distance to $\mathrm{RN} j$ and $l_{n 0}^{2}=x_{n}^{i j 2}+y_{n}^{i j 2}$ is the squared distance to the BS. We repeat the operation for all $j$ and we collect the obtained RN power values in a set $\mathcal{P}_{i}=\cup_{j}\left\{\tilde{P}_{R N}\left(p_{n}^{i j}\right)\right\}$.

3) We compute: $P_{R N}^{L}=\min \mathcal{P}_{i}, P_{R N}^{U}=\max \mathcal{P}_{i}$.

The accuracy of the approach improves with $N$. Given that the bounds are usually tight, we propose to approximate $P_{R N}^{*}$ by: $P_{R N}^{*} \approx\left(P_{R N}^{L}+P_{R N}^{U}\right) / 2$. Hence, using this approximation, we do not need to compute integral (9) for several values of $P_{R N}$. Our approach is summarized in Algorithm 1.

\section{Numerical Results}

\section{A. Simulation Settings}

Two alternatives are compared for our proposal, which differ at step 4 of the algorithm: use either the fluid model ((4) and (11)) or the exact SIR formulation (2). 


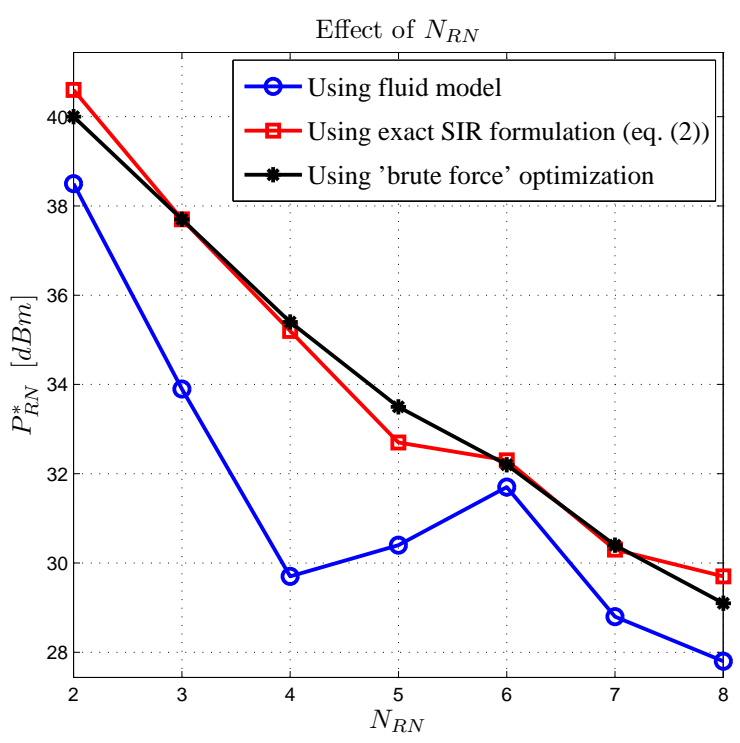

Fig. 3. Effect of $N_{R N}(d=3 / 8 D, \phi=0, \eta=3.5)$
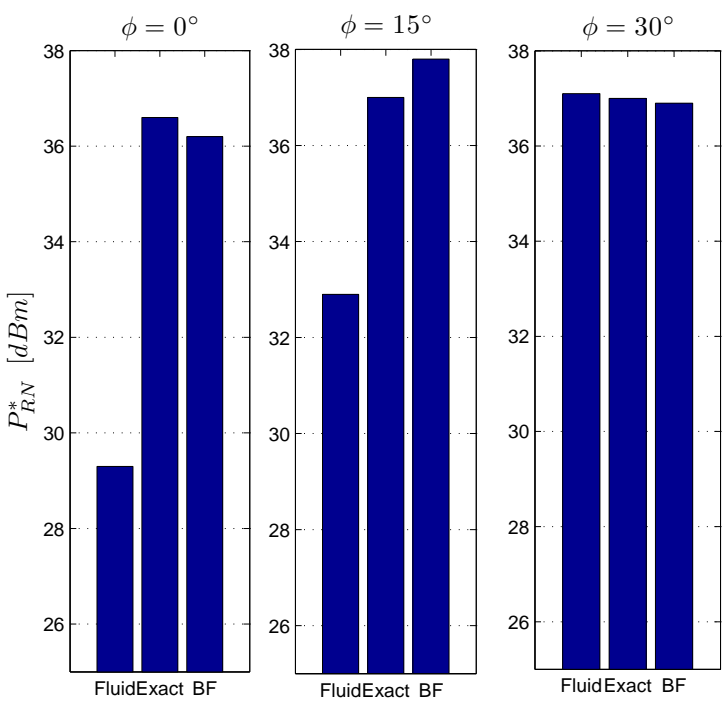

Fig. 4. Effect of $\phi\left(N_{R N}=3, \eta=3.5\right.$ and $\left.d=9 / 20 D\right)$

We consider a network of 4 rings of cells around a central cell. We set the inter-BS distance to $D=2 R_{B S}=$ $500 \mathrm{~m}$. Computations are performed on a grid of points of $\mathcal{A}_{c}$ of resolution $10 \mathrm{~m}$. We set $P_{B S}=46 \mathrm{dBm}$ [1]. Results are compared with the value of $P_{R N}^{*}$ obtained by means of brute force optimization: SIR and spectral efficiency are computed for each point of a grid of resolution equal to $10 \mathrm{~m}$, and for each considered $\mathrm{RN}$ power between 25 and $40 \mathrm{dBm}$ with a $0.1 \mathrm{~dB}$ step.

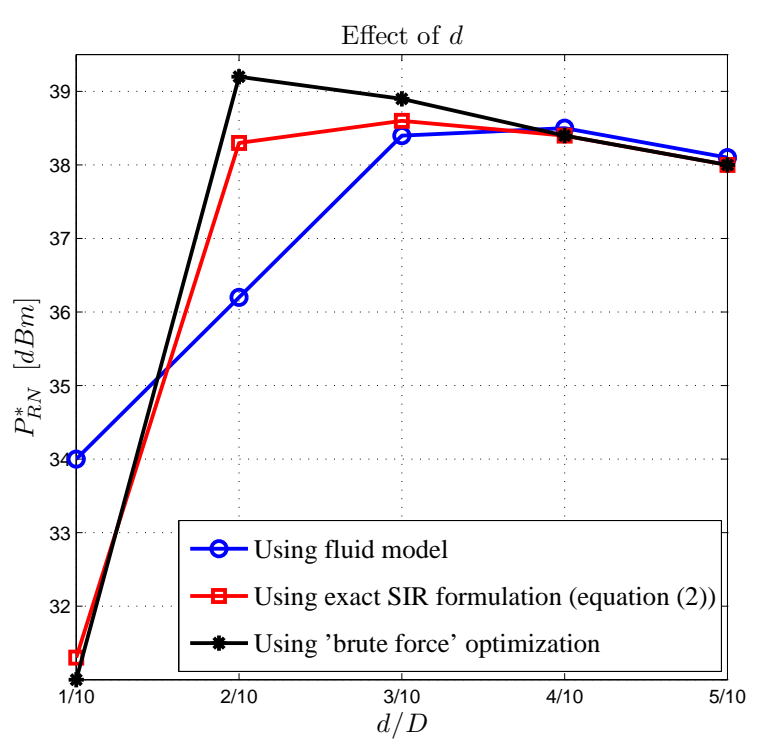

Fig. 5. Effect of $d\left(N_{R N}=3, \eta=3, \phi=30\right)$

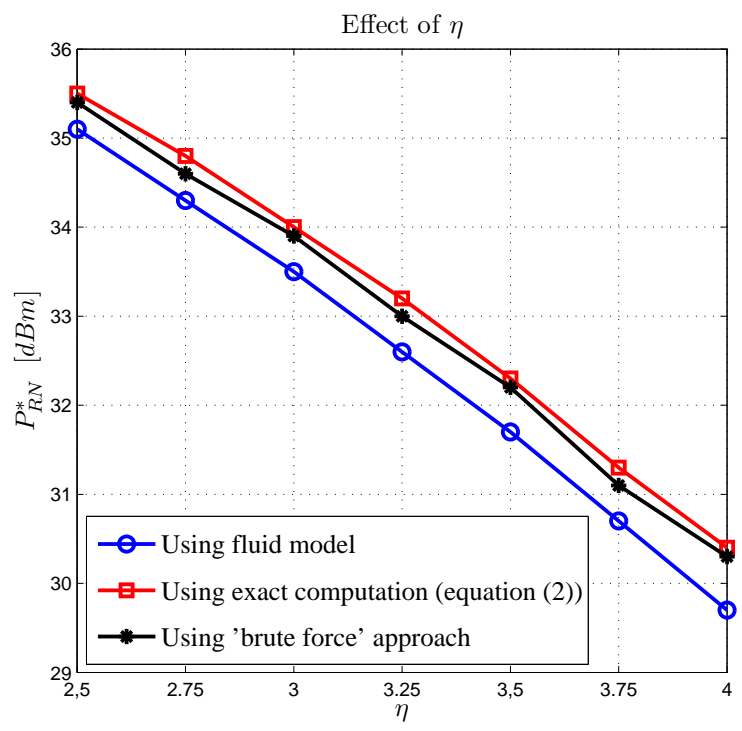

Fig. 6. Effect of $\eta\left(N_{R N}=6, d=3 / 8 D, \phi=0\right)$

\section{B. Results Analysis}

Figure 3 shows the effect of $N_{R N}$. As expected, $P_{R N}^{*}$ is a decreasing function of $N_{R N}$ because when the RN density increases, their SIR areas shrink. In all cases, the optimal power found using Eq. (2) is within $1 \mathrm{dBm}$ of the optimal (in most cases, even within $0.5 \mathrm{dBm}$ ). On Figures 4 and 5, we see that $\phi$ and $d$ have also little impact on the accuracy of $P_{R N}^{*}$. The fluid model provides faster results at the price of reduced accuracy. It is known that the fluid model is more accurate when the 
network is regular or close to be regular. This explains why results are better when $N_{R N}$ increases and when RNs are not too close from the BS. When $\phi=0$ and $d=9 / 20 D$, RNs from different cells are clustered close to the cell corners so that the obtained $\mathrm{RN}$ network is very irregular. In this case, the fluid model applied to our algorithm yields a very inaccurate result. The precision improves for growing $\phi$ because RNs tend to be more regularly spaced, and for $\phi=30 \mathrm{deg}$ the difference with the optimal value is approximately $0.2 \mathrm{dBm}$.

Figure 6 shows that $\eta$ has little influence on the accuracy of our approach. In the chosen scenario, the fluid model provides also a good approximation.

We have also performed simulations considering lognormal shadowing, and compared our approach with the result of Monte Carlo simulations, finding out that our algorithm is still within $0.3 \mathrm{dBm}$ of the optimal value even for shadowing standard deviation $\sigma=10 \mathrm{~dB}$.

Finally our algorithm is computationally faster compared to brute force optimization. The latter requires $O\left(N_{P} N B N_{R N}\right)$ operations. We notice that $\left|\mathcal{P}_{i}\right|$ is negligible compared to $N$ and to $B N_{R N}$, so that Algorithm 1 using (2) requires $O\left(N B N_{R N}\right)$ operations, while using (4) it requires $O\left(B N_{R N}+N\right)$ operations. We have also observed that $N$ can be kept sensibly lower in our algorithm, compared to brute force. As an illustrative example, using Matlab for the presented scenarios, the savings in terms of computational time are of several orders of magnitude (from few seconds for our algorithm to several hours for the brute force).

\section{CONCLUSION}

In this paper we have proposed a fast method to compute, for a given RN placement pattern, the RN transmit power optimizing the average cell spectral efficiency. This method is based on matching the SIR area of stations with their serving area, as closely as possible. Fluid model is proposed as a means for computing the SIR, because of the lower computational burden associated with it, as compared to straightforward SIR computation. However, the increased speed comes at the price of an accuracy loss. Results show that our method yields accurate results, particularly when an exact SIR formulation is adopted. However, accuracy is still high on a number of scenarios, when fluid model is used. The tradeoff between computational speed and precision is investigated. Future work may study the impact of bursty traffic and backhaul link on performance.

\section{REFERENCES}

[1] 3rd Generation Partnership Project (3GPP). TR 36.814 v9.0.0: Technical specification group radio access network; further advancements for E-UTRA physical layer aspects (release 9), Mar. 2010.

[2] N. Krishnan, R.D. Yates, N.B. Mandayam, and J.S. Panchal. Bandwidth sharing for relaying in cellular systems. Wireless Communications, IEEE Transactions on, 11(1):117 -129, january 2012.

[3] C. Bae and D.-H. Cho. "Fairness-Aware Adaptive Resource Allocation Scheme in Multihop OFDMA Systems". Communications Letters, IEEE, 11(2):134-136, Feb. 2007.

[4] Y. Cui, V.K.N Lau, and R. Wang. "Distributive subband allocation, power and rate control for relay-assisted OFDMA cellular system with imperfect system state knowledge". IEEE Trans. Wireless Commun, 8(10):5096-5102, Oct. 2009.

[5] Y. Hua, Q. Zhang, and Z. Niu. "Resource Allocation in Multi-cell OFDMA-based Relay Networks". In Proc. INFOCOM, pages 19, Mar. 2010.

[6] Nokia Siemens Networks. R1-106216 - Type-1 Relay Performance for Downlink. 3GPP TSG-RAN WG1 Meeting 63, Nov. 2010.

[7] O. Bulakci, A. Bou Saleh, S. Redana, B. Raaf, and J. Hamalainen. Flexible backhaul resource sharing and uplink power control optimization in LTE-advanced relay networks. In Vehicular Technology Conference (VTC Fall), 2011 IEEE, pages $1-6$, sept. 2011.

[8] J.-M. Kelif, M. Coupechoux, and P. Godlewski. Spatial outage probability for cellular networks. In Global Telecommunications Conference, 2007. GLOBECOM '07. IEEE, pages $4445-4450$, nov. 2007.

[9] M. Salem, A. Adinoyi, M. Rahman, H. Yanikomeroglu, D. Falconer, and Y.-D. Kim. Fairness-aware radio resource management in downlink OFDMA cellular relay networks. IEEE Trans. on Wireless Communications, 9(5), May. 2010.

[10] M. Pikhletsky, F. Khafizov, J. Zhang, and H. Zhuang. ”Dynamic Radio Resource Management for OFDMA-Based Relay Enhanced Cellular Network". In Proc. GLOBECOM, pages 1-5, Dec. 2011. 